\title{
Teen Vaping and the Delta Variant of COVID-19
}

\section{Kiersten Geary ${ }^{1}$ and Allen Fred Fielding ${ }^{2 *}$}

${ }^{1}$ Third Year Dental Student at Kornberg School of Dentistry, Philadelphia, Pennsylvania, USA

${ }^{2}$ Professor, Department of Oral and Maxillofacial Surgery, Kornberg School of

Dentistry, Philadelphia, Pennsylvania, USA

*Corresponding Author: Allen Fred Fielding, Department of Oral and Maxillofacial Surgery, Temple University Hospitals, Philadelphia, USA.
Received: September 20, 2021

Published: November 10, 2021

(C) All rights are reserved by Kiersten Geary and Allen Fred Fielding.

\begin{abstract}
The emerging delta variant of COVID-19 has shown different disease tropism from the previous alpha variant and is infecting young adults and teens at higher proportions. Additionally, teens today are utilizing e-cigarettes at alarmingly high rates. This is leading to EVALI (e-cigarette associated lung injury) in this population, which puts them at a higher risk if infected COVID-19. This study seeks to explore previous COVID-19 infections of patients with a history of cigarette use as a framework for prospective COVID-19 infections in patients with history of e-cigarette use and EVALI.
\end{abstract}

Keywords: COVID-19; EVALI; Delta Variant

\section{Introduction}

In 2018, the US Surgeon General declared e-cigarette use among youth to be an epidemic after a 78\% increase from 2017 to 2018 [1]. A 2019 study found that $27.5 \%$ of high school students used e-cigarettes [5]; later that year, the COVID-19 pandemic began in Wuhan, China. What started as the emergence of pneumonia symptoms in a single town evolved into a pandemic. During the early stages of COVID-19, it was found by Lui., et al. that patients with a history of smoking have an increase in disease severity by up to 14 times [7].

\section{Discussion}

At the start of the $21^{\text {st }}$ century, digitalization was everywhere including cigarettes. E-cigarettes emerged as a tobacco cessation product but as they gained popularity, dangers of their own came apparent. E-cigarettes a vaporize liquid that contains nicotine and flavoring agents became very popular. The addition of flavoring compounds to e-cigarettes makes them more marketable to teens than the traditional cigarette. Additionally, many e-cigarette users are teens and young adults; if these individuals develop a dependence for nicotine or THC, both of which can be used in e-cigarettes, they may have lung injuries, impeded brain development or develop lifetime dependence [8]. Though e-cigarettes may seem safer than traditional smoking, users still inhale smoke while using e-cigarettes, leading to potential lung damage (EVALI). In the teen and young adult population, EVALI has been a rising concern in those who use e-cigarettes. EVALI, or e-cigarette/vaping associated lung injury, is a reaction to a product in e-cigarettes; more research is needed to determine the exact compound. Presently the FDA (Federal Drug Administration) has put off its decision on 
e-cigarette safety that was due on a September 9, 2001 deadline. Presently some 500 companies have submitted applications for 6.5 million products [9]. As of February 2020, there have been almost 3,000 EVALI cases requiring hospitalization with over 65 deaths $[2]$.

Kaur., et al. discussed the scientific basis for smoker's increased susceptibility to COVID-19. The spike protein on the SAR-CoV2 utilizes the ACE2 receptor to invade respiratory epithelial cells; ACE2 receptor expression is upregulated in lung epithelia of smokers, increasing the likelihood of viral invasion. Additionally, the inflammatory response to smoke inhalation increases membrane permeability, which also increases likelihood of viral invasion [6]. While these findings were found primarily in those using traditional cigarettes, EVALI is thought to have a similar pathogenesis.

The alpha variant of SAR-CoV2 was seen to fatally affect individuals with an average age of 65 and fewer than $10 \%$ of cases in a study by Du., et al. were younger than 50 years old [5]. However, the emergence of the delta variant of SAR-CoV2 shows increased affinity for younger individuals. According to the American Academy of Pediatrics, children only represent $15 \%$ of all SAR-CoV2 cases but in a 2 week period from 08/26/21 to 09/02021, children represent over a quarter of the cases. There was variation in age ranges reported by each state with the upper limit ranging from age 14 to age 20 [3]. However, these age ranges are affected by the vaping epidemic so the data is still relevant, though incomplete, for this review.

\section{Conclusion}

With the increase in pediatric infection rates with SARs-CoV2 and the already prominent vaping epidemic, the young adult population is at a unique risk status. This population is not typically considered high risk and should be brough to the forefront of discussions about COVID-19 treatment. More research is needed to determine the best prevention and treatment modalities for this population.

\section{Bibliography}

1. Centers for Disease Control and Prevention. Surgeon General's advisory on e-cigarette use among youth. Centers for Disease Control and Prevention (2019).
2. Centers for Disease Control and Prevention. Outbreak of lung injury associated with the use of e-cigarette, or vaping, products. Centers for Disease Control and Prevention (2019).

3. Children and Covid-19: State-level data report. American Academy of Pediatrics (2021).

4. Cullen KA., et al. "e-Cigarette Use Among Youth in the United States, 2019". JAMA: The Journal of the American Medical Association 322.21 (2019): 2095-2103.

5. Du Y., et al. "Clinical Features of 85 Fatal Cases of COVID-19 from Wuhan. A Retrospective Observational Study". American Journal of Respiratory and Critical Care Medicine 201.11 (2020):1372-1379.

6. Kaur G., et al. "SARS-CoV-2 COVID-19 susceptibility and lung inflammatory storm by smoking and vaping". Journal of Inflammation Research 17.21 (2020).

7. Liu W., et al. "Analysis of factors associated with disease outcomes in hospitalized patients with 2019 novel coronavirus disease". Chinese Medical Journal 133.9 (2020): 1032-1038.

8. National Center for Chronic Disease Prevention and Health Promotion (US) Office on Smoking and Health. E-Cigarette Use Among Youth and Young Adults: A Report of the Surgeon General. Atlanta (GA): Centers for Disease Control and Prevention (US). Chapter 1, Introduction, Conclusions, and Historical Background Relative to E-Cigarettes (2016).

9. Maloney J. "FDA Puts Off Decision on Juul B3". Wall Street Journal (2021).

\section{Volume 5 Issue 12 December 2021 (C) All rights are reserved by Kiersten Geary and Allen Fred Fielding.}

